\title{
Vers une théorisation de l'approche géographique du tourisme
}

Introduction au dossier

Mathis Stock

\section{OpenEdition \\ Journals}

Édition électronique

URL : http://journals.openedition.org/tourisme/271

DOI : 10.4000/tourisme.271

ISSN : 2492-7503

Éditeur

Éditions touristiques européennes

\section{Édition imprimée}

Date de publication : 1 décembre 2010

Pagination : $19-23$

ISSN : 2109-5671

\section{Référence électronique}

Mathis Stock, "Vers une théorisation de l'approche géographique du tourisme », Mondes du Tourisme [En ligne], 2 | 2010, mis en ligne le 30 septembre 2015, consulté le 22 septembre 2020. URL : http:// journals.openedition.org/tourisme/271 ; DOI : https://doi.org/10.4000/tourisme.271

\section{(c) $(1) \&$}

Mondes du tourisme est mis à disposition selon les termes de la licence Creative Commons Attribution - Pas d'Utilisation Commerciale - Pas de Modification 4.0 International. 


\title{
INTRODUCTION AU DOSSIER
}

\section{Vers une théorisation}

\section{de l'approche géographique du tourisme}

\author{
Mathis STOCK
}

[mathis.stock@iukb.ch]

Professeur de géographie du tourisme à l'Institut universitaire Kurt Bösch (IUKB), Sion (Suisse), Centre de recherche interdisciplinaire sur le tourisme (Crit)

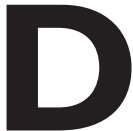
epuis trente ans, nous observons, en géographie comme d'ailleurs dans d'autres disciplines des sciences sociales, un engouement pour les questions de tourisme. Le travail effectué porte majoritairement sur la dimension empirique de la recherche scientifique, tandis que le travail conceptuel et méthodologique est moins investi. Nous nous trouvons alors devant un problème scientifique majeur : développer un ensemble de concepts reliés entre eux, robustes et pertinents, qui guident les chercheurs dans leur traitement des multiples phénomènes touristiques et qui permettent de les enchaîner dans l'argumentaire explicatif.

Nous avons, dans l'appel à communications pour le colloque de Sion, support du présent dossier ${ }^{(1)}$, identifié deux domaines de questionnement sur lesquels il nous semblait important de porter l'effort.
I Q Quels théories et modèles novateurs pour appréhender avec plus de pertinence le tourisme ? Cette question vise à identifier les outils conceptuels nouveaux, innovants, qui nous permettraient de saisir les phénomènes touristiques avec plus de pertinence qu'en l'absence de tels outils conceptuels. Qu'elles proviennent de la géographie ou d'ailleurs, il nous a semblé nécessaire d'axer le questionnement sur les innovations conceptuelles, et non seulement sur la poursuite de l'application de concepts éprouvés. En effet, on peut estimer que le niveau de langage acquis par les géographes jusqu'ici pour dire quelque chose de sensé sur le tourisme manque de subtilité( ${ }^{(2)}$. Par exemple, nous disposons seulement de quatre formes élémentaires de lieux touristiques : site, comptoir, station, ville (Knafou et al., 1997 ; Équipe Mit, 2002), certes affinées ensuite ( $c$. Duhamel, 2003) : est-ce que cela suffit pour appré- hender la diversité des lieux touristiques ? Nous disposons de quelques modalités de rapports du touriste à l'espace - le vide, le paysage, le sauvage, l'authentique, le patrimoine (MacCannell, 1999 ; Hennig, 1999 ; Équipe Mit, 2002), mais qui ne sont pas systématiquement conceptualisés. Nous disposons aussi de modélisations unidimensionnelles des stations touristiques - en fait "tourism area life cycle" (Butler, 1980) - sans que les ressorts du changement soient clairement identifiés (cf. Shaw et Williams, 1997). Ce ne sont là que quelques exemples qui montrent qu'il manque un vocabulaire plus différencié, d'une part, et des modèles plus stabilisés qui guident l'investigation, d'autre part.

2 - Quelles implications la prise en compte du tourisme a-t-elle pour les théories et modèles en géographie ? Cette question vise à identifier un "retour" des découvertes dans le champ du tourisme vers les théo- 
ries géographiques, et peut s'exprimer de la façon suivante : on peut supposer que, du fait de l'injection de phénomènes touristiques dans les modèles et théories classiques de la géographie, ces derniers ne fonctionnent plus. C'est clairement le cas du modèle des lieux centraux, mais aussi des modèles sur la mobilité dont les migrations et les mobilités quotidiennes étaient les seuls abordés ; on songe aussi aux travaux sur les "identités spatiales" : les référents géographiques de l'identité n'ont pas pris en compte le processus d'identification à l'œuvre à travers les déplacements touristiques. On peut alors défendre la thèse suivante : la prise en compte du fait touristique rend inopérants ou, au mieux, nécessite d'amender les théories et modèles en géographie. C'était de fait la question que posait Olivier Lazzarotti dès 2003 : "la géographie peut-elle s'agréger à un tel phénomène, l'ériger en objet propre et le traiter sans elle-même se remettre (...) en cause?" (Lazzarotti, 2003, p. 262). Y répondre est à l'évidence un défi immense pour les chercheurs : il s'agit de cerner l'apport des phénomènes touristiques pour la modélisation en géographie; d'une part et de proposer à terme de nouvelles "modélisations avec tourisme”, d'autre part.

\section{THÉORIE, MODÈLE, CONCEPT}

À quoi sert la théorie, un modèle, un concept ? Quelle est la fonction de la théorie dans le travail scientifique ? On peut définir "théorie" de multiples façons : selon Herbert Simon (2004), une théorie désigne un "système arborescent de concepts". Il s'agit d'un ensemble conceptuel qui définit une certaine perspective et qui aide à sélectionner, dans le flux des événements, les éléments pertinents pour la description, l'analyse, l'interprétation et l'explication. Définie ainsi comme une perspective, la théorie est une construction symbolique qui vise à donner sens aux multiples faits qui se présentent à nous. C'est ainsi, entre autres, que l'on peut comprendre le mot de Bruno Latour (1996) : "les faits sont faits", c'està-dire construits, entre autres, à l'aide d'un appareillage conceptuel.

Ainsi, il ne s'agit pas seulement d'un "ensemble cohérent d'énoncés à visée scientifique” (Ruby, 2003, dans Lévy et Lussault), c'est-à-dire un corpus fermé d'énoncés qui, en généralisant et en faisant abstraction d'éléments, réduit la complexité des phénomènes touristiques. Au contraire, la théorie devient un outillage conceptuel, toujours au travail, toujours mobilisable et mobilisé dans l'action d'observation mise en ouvre par les chercheurs. Cela a trois implications. Primo, "théorie" désigne un élément du monde symbolique qui guide les chercheurs dans leurs investigations empiriques et oriente le processus cognitif. C'est un garde-fou contre les conceptualisations ad hoc, empreintes d'arbitraire. Secundo, si l'on appréhende la théorie dans une perspective temporelle de l'activité de recherche, la théorie devient, pour le chercheur, un moyen de prédiction. À un temps t1, le chercheur élabore ou s'approprie une théorie, à un temps $\mathrm{t} 2$, il en déduit ce que le monde devrait être si la perspective était juste. Il prédit donc ce qu'il va tester en $\mathrm{t} 3$, à savoir l'adéquation de la réalité empirique avec la théorie. Tertio, la théorie donne une perspective sur les enchaînements de faits empiriques, elle donne sens, par exemple, aux multiples corrélations, notamment d'ordre statistique, que l'on peut mettre en évidence. Cependant, la variabilité des faits empiriques et des enchaînements n'est pas prise en charge par la théorie. Celle-ci n'est donc pas "englobante", au sens où elle intègrerait l'ensemble des énoncés portant sur le monde phénoménal, mais elle propose le sens du lien entre ces éléments.

Il s'agit donc d'une visée d'intelligibilité du monde, par une opération de sélection et d'agencement des faits que propose la théorie. Pour cela, elle s'appuie sur la mise en relation de concepts.

Quel est le rapport entre "théorie" et "modèle" ? Les modèles sontils déduits des théories ou mettentils en forme la théorie ? Selon les disciplines, "modèle" s'entend différemment ( $f$.Nouvel, 2002) et oscille entre deux acceptions. L'une est proche du "type idéal" (Idealtyp) de Max Weber, et décrit un ensemble de concepts parcimonieux et abstraits permettant de mesurer la dis- 
tance avec les faits empiriques que l'on peut "injecter" dans le modèle (Weber, 1922). La seconde se rapproche du modèle tel qu'il est défini par les économistes, servant à désigner la synthèse effectuée de la réalité, à l'aide d'éléments essentiels. Le modèle remplace donc la réalité, mais ne sert plus à l'observer et, éventuellement, à modifier le modèle. Si l'on veut garder intacte la fonction heuristique, il semble plus judicieux de garder le sens du modèle comme étalon de mesure par rapport à la réalité. Par exemple, le type idéal de l'exotisme ou de la station touristique pourrait être utilisé pour mesurer la distance avec ce que l'on peut observer empiriquement.

Enfin, la notion de "concept" est également à réfléchir. Êtant donné que nous formulons des problèmes (Popper), que nous construisons la réalité à l'aide de concepts (Bachelard), le travail sur les façons de concevoir ce monde symbolique est crucial. Comment conceptualiser ? Par analogie ? Est-ce que les métaphores sont un frein à la conceptualisation ou sont-elles un passage obligé ? Peut-on se passer du concept au profit des images qui sont produites ? Le philosophe Ernst Cassirer (1927) distingue deux types de concept : concepts substantiels et concepts relationnels. Ces derniers permettent de classer sans devenir eux-mêmes une classe, ou indiquent une fonction dans le monde du tourisme sans décrire un élément par abstraction ou comparaison a priori. Ils décrivent une relation ; ce sont des concepts relationnels. Ce sont les plus difficiles à maîtriser, car il s'agit d'éléments qui ne sont pas nécessairement nommés en tant que tels dans le langage commun ou qui sont nommés en tant que "choses". Il faut créer le mot en même temps que l'on crée la fonction dans une chaîne d'événements. Ainsi, les concepts ne sont pas des généralisations d'éléments substantiels, mais enchaînent plusieurs éléments dans une relation. On pourrait, par exemple, conceptualiser le tourisme ou le touristique comme une relation spécifique au monde.

Quelle est donc la fonction de ces éléments dans l'observation scientifique ? Dans l'ensemble, et sans tenir compte de leurs différences, la théorie, le modèle, le type idéal, le concept sont des outils qui guident le chercheur dans l'observation du monde. Ils permettent un détachement, une prise de distance par rapport au monde dans lequel sont enchâssés les chercheurs. Ce détachement étant plus ou moins contrôlé, les théories ou modèles aident à prendre des décisions, à formuler des jugements en délimitant ou en enchaînant des faits dans un certain sens. Ils permettent de faire des choix par rapport à ce que le chercheur appréhende et par rapport à ce qu'il laisse de côté. Il peut opérer des sélections qui sont non arbitraires, car argumentées à l'aide d'un référent qu'il est possible de remettre en cause. Il s'agit à chaque fois d'une perspective spécifique sur le monde - qui exclut d'autres perspectives au moment de leur utilisation - qui aide le chercheur à cheminer dans les innombrables faits qui se présentent à lui. Il est intéressant d'observer qu'en proposant des perspectives spécifiques, les théories et modèles sont ainsi incommensurables, car a priori incompatibles entre eux.

\section{COMMENT CONCEPTUALISER LES MULTIPLES PHÉNOMÈNES DU TOURISME ?}

Si la théorie aide à développer une perspective cohérente sur le monde, la question se pose : que peut-on théoriser ? Peut-on faire une théorie du tourisme ? Peut-on faire une théorie géographique du tourisme ? Convient-il d'élaborer une théorie de la circulation dans laquelle le tourisme prend place ou encore une théorie de l'urbain, de l'identité, de la pratique, de la spatialité etc. dans laquelle le tourisme prend place ? La réponse à cette question dépend sans doute de la façon dont on définit l'objet de recherche "tourisme".

Elle est très intéressante à poser, car l'on voit à l'œuvre des choix différents qui se présentent aux chercheurs quant à l'ambition théorique. Si le concept "tourisme" définit un système d'acteurs, de pratiques et de lieux visant à la re-création par le déplacement et l'habiter temporaire de lieux autres, on dispose d'une certaine perspective qui permet d'observer des assemblages dif- 
férents par rapport à des définitions portant sur le tourisme comme "secteur économique". Ainsi, la théorie porterait sur un ensemble d'éléments identifiés comme objet substantiel selon la classification de Cassirer. Mais, le tourisme, au lieu d'être défini comme système, pourrait aussi être défini comme "intentionnalité" ou "registre d'action" ou "régime d'engagement", à l'instar de ce que Boltanski et Thévenot (199|) décrivent comme des "cités" dans le discours de justification, et de ce que John Urry (1990) appelle "regard touristique", bref, ce qu'on appelait "sens" chez Max Weber (1922) ou Alfred Schütz (1973). Dans ce cas, "tourisme" ne se référerait pas à un monde social autonome ou à un "système" défini par un ensemble énumérable d'actants, mais plutôt à un ensemble de règles ou de codes qui guident les pratiques des touristes, des opérateurs économiques et de l'État et d'autres collectivités et qui donnent lieu à des façons de se mouvoir, de sentir, de donner sens aux activités qui sont différentes par rapport à d'autres régimes d'engagement. On aurait ainsi un objet de recherche qui se définirait par son caractère relationnel. La théorie porterait sur les manifestations multiples du "touristique”, plutôt que sur un objet autonome.

On dispose alors de plusieurs possibilités de conceptualisation, qui dépendent des différents objectifs de théorisation. La théorisation comme processus de construction d'une théorie sur les éléments touristiques des sociétés humaines est multiple; il n'y a pas de voie unique. Peut-être justement convient-il de ne pas viser une théorie du tourisme, mais plutôt un modèle du tourisme comme rapport au monde au sein d'une théorie de la société ou du processus de civilisation ou de l'habiter.

\section{PENSER \\ GÉOGRAPHIQUEMENT LE TOURISME}

Penser géographiquement les phénomènes touristiques et proposer des éléments de théorisation de l'approche géographique du tourisme. Tel est l'enjeu de ce dossier. Plusieurs contributions ont été sélectionnées afin de montrer comment un progrès conceptuel peut être bénéfique, en ce qu'il donne un moyen explicatif plus adéquat.

D'abord, un article portant sur la méthodologie d'ordre visuel afin de comprendre comment les habitants temporaires que sont les touristes imaginent/imagent la station touristique. Émeline Hatt y propose une réflexion méthodologique et une mise en œuvre empirique, à travers l'exemple de Seignosse, afin d'évaluer dans quelle mesure la participation des touristes, et non seulement des résidents, aux procédures d'aménagement a des effets bénéfiques sur la qualité de l'urbanisme des stations. Si cet article pouvait inspirer d'autres chercheurs à proposer des contributions d'ordre méthodologique, nous gagnerions en moyens d'accès aux phénomènes touristiques.

Amandine Chapuis propose ensuite une réflexion sur le concept de performance qui pourrait être utilisé pour décrire les pratiques touristiques. Pour ce faire, elle construit un passage possible de l'utilisation de ce terme de la performance comme métaphore à la performance comme concept pouvant servir à décrire la façon dont les pratiques touristiques se déroulent, mais aussi sur la construction de l'espace touristique par des performances répétées. Nous sommes là dans le registre de la spatialité, c'est-à-dire dans le rapport à l'espace qui est, en géographie, une problématique de plus en plus “investiguée”. Une analyse géographique plus approfondie des pratiques touristiques est en jeu.

Travailler le lien entre urbanité et tourisme est une tentative ancienne en géographie, à travers le "tourisme urbain". Vincent Coëffé propose une investigation originale qui remet en cause les façons classiques de penser l'urbain par le touristique. En construisant la notion d'urbanité et en l'articulant aux mobilités touristiques, il est capable d'appréhender les multiples différentiels d'urbanité où se loge le tourisme, et ne restreint pas l'analyse à seulement un géotype, la ville. Travaillant la spatialité dans ses multiples modalités - pratiques, normes, valeurs, imaginaires - l'analyse est centrée sur l'acteur indivi- 
duel - il permet un lien intéressant avec l'article d'Amandine Chapuis où le faire du touriste est mis au centre de l'investigation.

S'appuyant également sur une analyse des spatialités, Anthony Goreau-Ponceaud porte son regard sur les pratiques de mobilités de la diaspora tamoule, de plus en plus informée par la re-création. Nous sommes là en présence d'une modalité spécifique de pratiques de recréation avec mobilité de la part de personnes dont les circulations à l'échelle mondiale entre les différents endroits de la diaspora font acquérir un capital spatial spécifique. Mobilisant des éléments identitaires, de construction de la famille et de re-création, ce type de mobilité constitue un assemblage spécifique, par rapport à d'autres pratiques de mobilité davantage investiguées. Cette contribution illustre le fait que le "touristique" ne se loge pas nécessairement dans un système social autonome, mais est présent dans de multiples pratiques (déplacement), rapports au monde (identité) et institutions (famille).

Ainsi, ces différents articles, qui identifient les multiples façons dont le "touristique" est mobilisé dans les situations et dont il est relié à d'autres éléments des sociétés humaines, contribuent à une conceptualisation renouvelée du tourisme. De l'analyse géographique du tourisme en tant que substance, on passe bien à une analyse géographique du tourisme comme relation.
( I) Ce dossier est issu du colloque Théorie, tourisme, géographie, qui s'est tenu à Sion du 15 au 17 juin 2009 pour le compte de la Commission de géographie de tourisme et des loisirs du Comité national français de géographie.

(2) L'observation que faisait Norbert Élias me semble importante afin de comprendre quelle tâche nous est assignée: "Nos langues sont ainsi faites que nous ne pouvons bien souvent exprimer un mouvement constant, une transformation continue, qu'en leur conférant d'abord par le lan-

\section{RéfÉRENCES BIBLIOGRAPHIQUeS}

Gaston BACHeLARD, La Formation de l'esprit scientifique, Vrin, 1993 (I 'réd. 1938).

Luc BoltANSKI et Laurent THEVENOT, De la justification. Sur les économies de la grandeurs, Gallimard, 199।.

Richard BUTLER, "The conception of a tourist area cycle of évolution: implications for management of resources", Canadian Geographer, vol. 21, n I, pp. 5-12, 1980.

Ernst CASSIRER, Philosophie symbolischer Formen, 1923-1929 (3 volumes), 1927.

Philippe DuHAMEL, "Les lieux touristiques", dans Mathis STOCK (coord.), Le Tourisme. Acteurs, lieux et enjeux, Belin, 2010 (3éd.). Norbert Elas, Qu'est-ce que la sociologie?, éd. de l'Aube, 199| (Ire éd. 1953).

Christoph HeNNIG, Reiselust. Touristen, Tourismus und Urlaubskultur, Suhrkamp, 1999. Rémy KNAFOU, Mireille BRUSTON, Florence Deprest, Philippe DuHAMEL, Jean-Christophe Gay et Isabelle SACAREAU, "Une approche géographique du tourisme", L'Espace géographique, vol. 26, n³, 193-204, 1997.

Bruno LATOUR, Petites leçons de sociologie des gage et la pensée le caractère d'un objet isolé, statique, et qu'ensuite seulement l'adjonction d'un verbe exprime le caractère mouvant de cet objet. Lorsque nous nous tenons par exemple au bord d'une rivière et que nous voulons conceptualiser l'écoulement continu des eaux et en parler, nous ne pensons pas et nous ne disons pas : 'Regarde l'écoulement continu de l'eau', mais nous pensons et disons: 'Regarde comme la rivière coule vite.' Nous disons 'le vent souffle', comme si le vent était autre chose qu'un souffle, comme s'il pouvait exister un vent qui ne souffle pas" (Qu'est-ce que la sociologie? pp. |32-133).

sciences, Le Seuil, 1996.

Olivier LAZZAROTII, "Tourisme et géographie : le grand dérangement", dans Mathis STOCK (coord.), Le Tourisme. Acteurs, lieux et enjeux, Belin, 2003 (3e éd, 20 I0).

Dean MACCANNeLL, The Tourist A New Theory of the Leisure Class, Schocken, 1999 (Ire éd. 1976).

Équipe MIT, Tourismes I. Lieux communs, Belin, 2002.

Pascal Nouvel (dir.), Enquête sur le concept de modèle, Puf, 2002.

Karl POPPER, Conjectures et réfutations. La croissance du

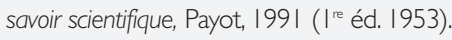

Christian RUBY, "Théorie", dans Jacques LÉVY et Michel LusSAULt (dir.), Dictionnaire de la géographie et de l'espace des sociétés, Belin, 2003.

Alfred ScHütz, Der sinnhafte Aufbau der sozialen Welt, Suhrkamp, 1973 (Ire éd. 1932).

Herbert SIMON, Les Sciences de l'artificiel, Gallimard, 2004 (Ire édition 1969).

Gareth SHAW et ALLAN Williams (dir), The Rise and Fall of British Coastal Resorts: Cultural and Economic Perspectives, Thomson, 1997.

John URRY, The Tourist Gaze, Sage, 1990.

Max WEBER, Wirtschaft und Gesellschaft. Grundriss der verstehenden Soziologie, Mohr, 1976 (Ire éd. 1922). 